\title{
EDITORIAL
}

\section{Preterm VLBW infants: post-extubation respiratory support}

\section{Journal of Perinatology (2006) 26, 449-451. doi:10.1038/sj.jp.7211542}

Prolonged intubation in preterm neonates is associated with increased morbidity, including greater infection risk and development of bronchopulmonary dysplasia (BPD). Very low birth weight (VLBW, $<1500 \mathrm{~g}$ ) infants often require ongoing respiratory support after extubation due to residual lung disease and/or apnea of prematurity. In recent years, there has been a growing trend toward early extubation after surfactant or complete avoidance of intubation and mechanical ventilation for preterm neonates. ${ }^{1,2}$

NCPAP has been effective for VLBW infants post-extubation because it recruits alveoli, stabilizes functional residual capacity (FRC), and maintains lung volumes. ${ }^{3,4}$ Anatomical issues predispose an infant to upper airway obstruction during spontaneous breathing, and CPAP may have a splinting effect on the upper airway. ${ }^{5}$ NCPAP also stabilizes a neonate's highly compliant chest wall by increasing the end-expiratory lung volume, thus improving pulmonary mechanics. ${ }^{3}$ Clinically, however, NCPAP can be difficult to maintain in proper position in VLBW infants, and when dislodged is rendered less effective as well as physically irritating. Complications of NCPAP in VLBW infants include nasal occlusion, nasal deformities, or pressure necrosis of the nasal septum. ${ }^{6}$

Nasal cannulas (NC), also used to support the breathing of recovering $\mathrm{VLBW}$ infants, are an effective means to deliver supplemental oxygen. Standard or low-flow NC therapy has been limited by inadequate humidification and the drying effect on upper airway mucosa, and for infants has a variably defined upperlimit of $0.5-21 /$ min (l.p.m.). ${ }^{7,8}$ Nasal mucosal injury and bleeding have also been associated with unheated NC flow (1 l.p.m.), although overly aggressive suctioning of the nares may contribute as well. ${ }^{9}$ Despite these issues, NC use is desired by pediatric caregivers because of the ease of caring for infants without interrupting oxygen therapy.

Vapotherm 2000i (Vapotherm Inc., Stevensville, MD, USA) is a relatively new respiratory device that delivers markedly higher $\mathrm{NC}$ flows in infants, in the range of 1-81.p.m. Company literature states that the membrane vapor exchange system humidifies the gas to $\sim 95 \%$ relative humidity and heats the oxygen/air mixture so that it is delivered at near body temperature. ${ }^{10}$ With the moisture content and temperature of the gas mixture comparable to that of an infant's upper airway, this theoretically minimizes desiccation of these delicate tissues. In adults, Vapotherm 2000i has been effective in decreasing hypoxemia and work of breathing for patients with chronic obstructive pulmonary disease and asthma with severe rhinitis. ${ }^{11,12}$ In the infant population, this device has not been rigorously studied, and with the exception of a few case reports, a recent observational study, and the company website, little information has been available. In this issue of the Journal, two reports expand this literature.

Woodhead et al. report the results of a small, randomized trial comparing the use of Vapotherm HFNC with standard high-flow NC (Std NC) in 30 newly extubated preterm neonates. They found that Vapotherm performed better than Std NC on the basis of a significantly lower respiratory effort score, more normal-appearing nasal mucosa on blinded exams, and less extubation failures. This pilot study is among the first prospective, randomized trials to evaluate the use of Vapotherm in newly extubated preterm neonates, and these initial results are encouraging. Unique to this study was use of an apparently new nasal mucosal exam score that did lend some objectivity to these observations, although validation of this tool between observers is unclear. The range of gas flow used in the Vapotherm group was 3.1 $1 \pm 0.61$.p.m., almost twice that of Std NC. Intrathoracic pressures were not assessed, thus the question remains regarding whether higher NC flows induce CPAPlike effects that impact results. Although the sample size was small, importantly no pneumothoraces were reported. Observer bias by the bedside clinical team could not be completely eliminated, which the authors acknowledge could have affected the respiratory effort score. Similarly, this bias may have impacted the crossover and reintubation rates, weakening the differences between groups. Despite these open-ended questions, we now have pilot data on the effects of Vapotherm HFNC vs Std NC therapy in a small, relatively mixed group of preterm infants upon extubation. In future studies, similar comparisons with a NCPAP group, longer duration of study interventions, and a focus on VLBW infants will be of interest. In addition, a broader range of Vapotherm flows should be evaluated, given that flow ranges used clinically are likely highly variable among centers.

The second study by Saslow et al. studied the work of breathing (WOB) in 18 preterm infants, contrasting the effects of Vapotherm at flows of 3, 4 and 5l.p.m. with continuous-flow NCPAP at $6 \mathrm{~cm}$ $\mathrm{H}_{2} \mathrm{O}$ (CPAP6) in each baby. There were no significant differences in WOB (inspiratory, elastic, or resistive), tidal volumes, or respiratory rates between all conditions. The unmeasured 'CPAP-effect' of HFNC, a common concern, was addressed with use of esophageal pressure $\left(P_{\mathrm{es}}\right)$ measurements to calculate an estimate of airway end-distending pressures. They found an increase in end-distending 
pressure from baseline (i.e. no support) at $\mathrm{CPAP} 6$ and at all three Vapotherm settings, with a mean increase of $<2 \mathrm{~cm} \mathrm{H}_{2} \mathrm{O}$. However, these pressure changes were markedly overlapped, and only reached statistical significance for Vapotherm at 51.p.m. vs CPAP6 $(P<0.03)$. One might expect to find a greater difference in pressures with increasing HFNC flows. ${ }^{8,13}$ This was not the case in the Saslow study, likely due to the small sample size, mild respiratory disease, and the heterogeneous group of preterm babies enrolled. Pulmonary compliance was mildly improved for Vapotherm at 5 l.p.m. vs CPAP6 $(P<0.03)$. Yet there was also a trend toward increased asynchrony with Vapotherm that may negate the compliance effect, especially with longer duration therapy. In this study, NCPAP was provided by a continuous flow source. In future studies, comparison of Vapotherm to variable-flow NCPAP will be of particular interest, as it has been shown that variable-flow NCPAP reduces the WOB and provides better lung recruitment compared to continuous flow NCPAP ${ }^{1,3}$ Further evaluation of pulmonary mechanics in preterm infants on Vapotherm HFNC therapy is clearly warranted, to better define its safety and efficacy.

These two small, randomized trials are a good first step in providing objective data regarding HFNC therapy with Vapotherm. The concept of providing heated, humidified gas therapy to minimize tissue injury and for improved comfort and tolerance by patients makes sense. The ability to do so via a NC for VLBW infants in particular makes the care, feeding and handling of these young patients simpler for caregivers and parents compared with NCPAP. These two studies by Saslow et al. and Woodhead et al. lend support to the common belief that HFNC may be as efficacious as NCPAP following extubation of VLBW infants.

Two major concerns still exist surrounding use of the Vapotherm HFNC in the neonatal population: (1) the unmeasured CPAP effect, and (2) the potential for infection, with exposure to Ralstonia spp. In 1993, Locke at al raised concern about the delivery of unregulated positive distending pressures (PDP) via NC gas flows, particularly associated with $0.3 \mathrm{~cm}$ diameter $\mathrm{NC}$ prongs but not with $0.2 \mathrm{~cm} \mathrm{NC}$ prongs. ${ }^{13}$ Subsequently, Sreenan et al. compared HFNC with NCPAP, measuring end expiratory $P_{\text {es }}$ in each patient to adjust HFNC gas flow (range 1-2.5 l.p.m.) to deliver pressure equivalent to NCPAP at $6 \mathrm{~cm} \mathrm{H}_{2} \mathrm{O}$. While the NC diameters were not reported, they found that in preterm infants $<2 \mathrm{~kg}$ HFNC at relatively common flows do deliver CPAP-equivalent PDP. ${ }^{8}$ More recently, a bench study showed Vapotherm to have higher pressures and resistance than NC or NCPAP, with a potential negative effect on WOB. ${ }^{14}$ In a recent commentary, Finer expressed concern regarding HFNC use (at flows $>1-2$ l.p.m.) and lack of knowledge of actual CPAP levels being delivered. ${ }^{15}$ The Saslow study is the first to address the question of pressures associated with Vapotherm, using $P_{\text {es }}$ measurements and calculation of end-distending pressures, and these data begin to support the equivalence of HFNC therapy with NCPAP.
Regarding the second issue, the CDC is in the midst of an investigation of the association of Ralstonia with Vapotherm devices, which first became public in October $2005 .{ }^{16}$ The source of Ralstonia remains unclear at present, but the bacteria have persisted despite revisions in cleaning procedures. ${ }^{17}$ Coincident with the 20 December 2005 public health notification by the CDC and FDA, Vapotherm Inc., announced a voluntary recall of all Vapotherm 2000i devices for enhanced disinfection procedures and revision of use recommendations. The FDA has advised use of alternative devices while the contamination source is being elucidated.

The phenomenon of use of an unproven modality in neonatology is not new, and the rapid, widespread use of Vapotherm is another example of this. The subjective impression that infants are more comfortable on Vapotherm HFNC and that they apparently tolerate it well does not outweigh the potential risks not yet fully understood. We can speculate that Vapotherm, with its heated and highly humidified gas mixture, may become a valuable respiratory support device for the neonatal population. Once the infectious concerns related to Vapotherm are resolved, this device or similar next generation devices may be cautiously brought back into clinical use for neonates and young infants. At that point, it is imperative that larger randomized, controlled trials be launched to provide reliable data on the safety, efficacy, and optimal timing of Vapotherm HFNC therapy. Future trials may also delineate which modalities, that is HFNC, NCPAP or something else, offer the best non-invasive respiratory support for infants, especially for VLBW infants upon extubation.

\section{Acknowledgments}

I thank my colleagues Lawrence M Nogee and Catherine $\mathrm{H}$ Jones for their valuable discussions and feedback regarding this editorial.

Maureen M Gilmore ${ }^{1,2}$
${ }^{1}$ Department of Neonatology, Johns Hopkins Bayview Medical
Center, Johns Hopkins University School of Medicine,
Baltimore, MD, USA and ${ }^{2}$ Department of Pediatrics,
Division of Neonatology,
Johns Hopkins University School of Medicine,
Baltimore, MD, USA
E-mail: mgilmor1@jhmi.edu

\section{References}

1 Pandit PB, Courtney SE, Pyon KH, Saslow JG, Habib RH. Work of breathing during constant- and variable-flow nasal continuous positive airway pressure in preterm neonates. Pediatrics 2001; 108(3): 682-685.

2 De Paoli AG, Morley C, Davis PG. Nasal CPAP for neonates: What do we know in 2003? Arch Dis Child Fetal Neonatal Ed 2003; 88(3): F168-F172.

3 Courtney SE, Pyon KH, Saslow JG, Arnold GK, Pandit PB, Habib RH. Lung recruitment and breathing pattern during variable versus continuous flow nasal continuous positive airway pressure in premature infants: An evaluation of three devices. Pediatrics 2001; 107(2): 304-308. 
4 Richardson CP, Jung AL. Effects of continuous positive airway pressure on pulmonary function and blood gases of infants with respiratory distress syndrome. Pediatr Res 1978; 12(7): 771-774.

5 Miller MJ, DiFiore JM, Strohl KP, Martin RJ. Effects of nasal CPAP on supraglottic and total pulmonary resistance in preterm infants. $J$ Appl Physiol 1990; 68(1): 141-146.

6 Loftus BC, Ahn J, Haddad Jr J. Neonatal nasal deformities secondary to nasal continuous positive airway pressure. Laryngoscope 1994; 104(8 Part 1): 1019-1022.

7 Finer NN, Bates R, Tomat P. Low flow oxygen delivery via nasal cannula to neonates. Pediatr Pulmonol 1996; 21(1): 48-51.

8 Sreenan C, Lemke RP, Hudson-Mason A, Osiovich H. High-flow nasal cannulae in the management of apnea of prematurity: A comparison with conventional nasal continuous positive airway pressure. Pediatrics 2001; 107(5): 1081-1083

9 Kopelman AE, Holbert D. Use of oxygen cannulas in extremely low birthweight infants is associated with mucosal trauma and bleeding, and possibly with coagulase-negative staphylococcal sepsis. J Perinatol 2003; 23(2): 94-97.

10 Vapotherm inc.. website [homepage on the Internet]. Stevensville, MD: Vapotherm Inc. Available from: http//:www.vtherm.com.
11 Nammour H, Cortez FV, Mehta K, Loube D. High flow humidified nasal room air improves hypercapnia during wakefulness and sleep in severe COPD patients. Am J Resp Crit Care Med 1999; 159: A804.

12 Bamford OS, Mehta KD, Cortez F. Effects of breathing warm humidified air on pulmonary function in asthmatic patients with chronic rhinitis. Chest 1999; 216: 291S.

13 Locke RG, Wolfson MR, Shaffer TH, Rubenstein SD, Greenspan JS. Inadvertent administration of positive end-distending pressure during nasal cannula flow. Pediatrics 1993; 91(1): 135-138.

14 Chang GY, Cox CC, Shaffer TH. Nasal cannula, CPAP and Vapotherm: effect of flow on temperature, humidity, pressure and resistance. PAS 2005; 57 : 1231.

15 Finer NN. Nasal cannula use in the preterm infant: oxygen or pressure? Pediatrics 2005; 116(5): 1216-1217.

16 Centers for Disease Control and Prevention (CDC). Ralstonia associated with vapotherm oxygen delivery device - United States, 2005. MMWR Morb Mortal Wkly Rep 2005; 54(41): 1052-1053.

17 Centers for Disease Control and Prevention (CDC). Update: Public health notification regarding ralstonia associated with vapotherm respiratory gas administration devices - United States, 2005. MMWR Morb Mortal Wkly Rep Early Release 2005; 54 December 20, 2005. 